\title{
A Cutoff in the X-ray Fluctuation Power Density Spectrum of the Seyfert 1 Galaxy NGC 3516
}

\author{
Rick Edelson \\ Astronomy Department; University of California; Los Angeles, CA 90095-1562; USA \\ X-Ray Astronomy Group; Leicester University; Leicester LE1 7RH; United Kingdom \\ Eureka Scientific; 2552 Delmar Ave.; Oakland, CA 94602-3017; USA \\ and \\ Kirpal Nandra \\ NASA/Goddard Space Flight Center; Laboratory for High Energy Astrophysics; Code 662; \\ Greenbelt, MD 20771; USA
}

\begin{abstract}
During 1997 March-July, RXTE observed the bright, strongly variable Seyfert 1 galaxy NGC 3516 once every $\sim 12.8 \mathrm{hr}$ for 4.5 months and nearly continuously (with interruptions due to SAA passage but not Earth occultation) for a 4.2 day period in the middle. These were followed by ongoing monitoring once cvery $\sim 4.3$ days. These data are used to construct the first well-determined X-ray fluctuation power density spectrum (PDS) of an active galaxy to span more than 4 decades of usable temporal frequency. The PDS shows no signs of any strict or quasi-periodicity, but does show a progressive flattening of the power-law slope from -1.74 at short time scales to -0.73 at longer time scales. This is the clearest observation to date of the long-predicted cutoff in the PDS. The characteristic variability time scale corresponding to this cutoff temporal frequency is $\sim 1$ month. Although it is unclear how this time scale may be interpreted in terms of a physical size or process, there are several promising candidate models. The PDS appears similar to those seen for Galactic black hole candidates such as Cyg X-1, suggesting that these two classes of objects with very difforent luminosities and putative black hole masses (differing by more than a factor of $10^{5}$ ) may have similar X-ray generation processes and structures.
\end{abstract}

Subject headings: galaxies: active - galaxies: individual (NGC 3516) - galaxies: Seyfert - x-rays: galaxies

\footnotetext{
${ }^{1}$ Universities Space Research Association
} 


\section{Introduction}

X-ray variability can, in principle, provide strong constraints on the physical conditions in the centers of Active Galactic Nuclei (AGN). Early observations of rapid variability in Seyfert galaxies implied large luminosity densities, providing the first compelling argument for the standard black hole/accretion disk model (Rees 1984) and against starburst models (Terlevich et al. 1995). In some models, the X-ray source is the primary emission component, so X-ray variability may be the most direct way to probe the smallest accessible regions of the central engine.

Measurement of a "characteristic variability time scale" may allow determination of the source size, luminosity density, and black hole mass. Early attempts to do this with unevenly sampled data involved measuring the "e-folding time" (or the similarly defined doubling time), defined as the minimum observed value of $t_{1 / e}=\left|[d \ln F / d t]^{-1}\right|$. However, this quantity is unstable, depending strongly on sampling length and signal-to-noise ratio (Press 1978). There have also been claims for quasi-periodic oscillations in AGN (e.g., Papadakis \& Lawrence 1993, 1995, Iwasawa et al. 1998), but these have been disputed (e.g., Tagliaferri et al. 1996), and there is certainly no consensus that such behavior has been observed in any AGN.

The best way to measure the characteristic variability time scale, and to characterize AGN variability in general, is to determine the fluctuation power density spectrum (PDS). Because of its highly eccentric orbit, EXOSAT provided the most useful AGN light curves gathered until recently with its uninterrupted 2-3 day "long-looks." The EXOSAT long-look PDS had a usable dynamic range of about 1.7 decades of temporal frequency $\left(\sim 10^{-3}\right.$ to $2 \times 10^{-5} \mathrm{~Hz}$, or $\sim 10^{3}$ to $5 \times 10^{4} \mathrm{sec}$ ). The upper frequency limit is the smaller of the Nyquist frequency (half the inverse of the fundamental sampling period) and the frequency at which the variations are typically greater than the noise, and the lower limit corresponds to $\sim 1 / 3$ of the observation length (c.g., Press 1978). These PDS generally rise smoothly to longer time scales as a power-law $\left(P(f) \propto f^{a}\right.$, where $P(f)$ is the power at temporal frequency $f$ ), with no features or signs of periodicity. All were apparently consistent with a single form, so-called "red noise" because the high temporal frequency slopes were stcep ( $a \approx-1.5$; Green et al. 1993; Lawrence \& Papadakis 1993). A turnover (which would be identified as a characteristic variability time scale) must occur at some longer time scale or the total variability power would diverge, but these PDS were not able to detect it because of the lack of low temporal frequency coverage. Attempts have been made to constrain this cutoff by combining data from a number of unrelated observations (e.g., McHardy 1989). The most convincing application to date was for NGC 4151 (Papadakis \& McHardy 1995), which found evidence that the PDS was cut off at a time scale of $\sim 2$ weeks. These previous efforts utilized highly unevenly sampled data, however, and therefore required complex and non-standard techniques that were not able to directly determine the PDS, leaving the results

with considerable uncertainty. Here we present the results of more standard PDS analysis of a new body of near-evenly sampled data. 


\section{Observations}

It was only after EXOSAT's mission had ended that the importance of evenly-sampled data and simultaneously probing long and short time scales became apparent. All X-ray satellites launched since then have had low-Earth orbits, for which Earth occultation generally corrupted short-term light curves beyond recovery. However, even though it too had a low-Earth orbit, the launch of the Rossi X-ray Timing Explorer ( $R X T E$ ) opened up interesting, new possibilities. It's high throughput, fast slewing and flexible scheduling make it ideal for obtaining even sampling on long time scales. Most importantly for this project, it has a large "continuous vicwing zone," defined as a region of sky near the orbital pole for which a source is visible over a number of consecutive orbits without interruption due to Earth occultation. Furthermore, any source in such a region is also observable for at least some part of each orbit, at all times of the year. This makes it possible to evenly sample both long and short time scales with a single instrument.

\subsection{NGC 3516}

We searched this region of sky and found, by fortunate coincidence, that it contained NGC 3516. This bright $(B \approx 13$ ) object was one of the original Seyfert galaxies (Seyfert 1943). It is bright in the X-rays as well, with $F(2-10 \mathrm{keV}) \approx 1.3-8 \times 10^{-11} \mathrm{erg} \mathrm{cm}^{-2} \mathrm{sec}^{-1}$ (Ghosh \& Soundararjaperumal 1991; Kolman et al. 1993; Nandra et al. 1997a). It shows a complex spectrum, and a simple power-law plus cold absorption model is inadequate to describe it. There is strong evidence for further spectral complexity in this source, including a "warm absorber" (Kolman et al. 1993; Kriss et al. 1993; George et al. 1998), a broad, variable iron K $\alpha$ line (Nandra et al. 1997a,b) and probably also Compton reflection (Kolman et al. 1993; Nandra \& Pounds 1994). The X-ray continuum flux of NGC 3516 is strongly variable, as is its spectrum (Kolman et al. 1993; Kriss et al. 1993). The ultraviolet continuum flux and C IV absorption also show variations on time scales as short as a few days to a week (Voit, Shull \& Begelman 1987; Koratkar et al. 1997). Because it is so bright an strongly variable, and most importantly because of its favorable location in the sky, NGC 3516 was chosen for this experiment.

\subsection{Sampling Pattern}

The sampling pattern was optimized to take full advantage of the unique attributes of $R X T E$ to obtain variability information spanning time scales from minutes to months. NGC 3516 was observed once every $\sim 12.8 \mathrm{hr}$ (=8 orbits) for 124 epochs, then quasi-continuously for 4.2 days, then again once every $\sim 12.8 \mathrm{hr}$ for another 124 epochs. (Combining the periodic sampling with snippets from the continuous period yielded 256 nearly-evenly-sampled scheduled epochs.) These were followed immediately by observations once every $\sim 4.3$ days ( $=64$ orbits) that are planned to continue for the lifetime of the satellite. 
The goal was for the sampling to be as close as possible to strictly periodic on each of three progressively shorter and more densely sampled time scales. This experiment came much closer than any previous effort, although certain unavoidable perturbations must be noted. During the continuous sampling, the light curve was interrupted by SAA passage, and a few time-critical observations of other sources also caused short interruptions. The total time lost to all of these effects was $69 \mathrm{ksec}$ ( $19 \%$ of the total duration). The $12.8 \mathrm{hr}$ sampling was interrupted three times for a total of 10 epochs (3.9\%), the longest being 6 epochs when the satellite went into safe mode from May 31 06:24 to June $222: 24$. For the longest sampling, there were a total of 3 interruptions $(2.3 \%)$. The real sampling pattern was matched as closely as possible to the ephemeris. The mean deviation between the ephemoris and observation times was $0.36 \mathrm{hr}$ ( $2.8 \%$ of a fundamental cycle) for the medium time scale and $0.98 \mathrm{hr}(1.0 \%)$ for the long time scale sampling. (The short time scale data were by definition tied to the ephemeris with no deviation.)

The sampling parameters are summarized in Table 1. For each time scale (short, medium and long) for which there is a near-evenly sampled light curve, column 2 gives the range of observing dates, column 3 , the mean sampling interval, column 4 , the number of points, and column 5 , the usable temporal frequency range, respectively.

\section{Data}

\subsection{Data Reduction}

The RXTE Proportional Counter Array (PCA) consists of 5 collimated Proportional Counter Units (PCUs), sensitive to X-rays in a nominal 2-100 keV bandpass. Some further details of the PCU and its performance are given by Jahoda et al. (1996). Here only the PCA STANDARD-2 data are considered, which have a minimum of $16 \mathrm{sec}$ time resolution and full detector and layer identification. The first of the PCU layers is most sensitive to cosmic X-rays and therefore only those data are employed herein. PCU units 3 and 4 were occasionally turned off due to performance problems and here only data from three of the PCUs $(0,1$ and 2$)$ are used. The

Table 1. Sampling Parameters

\begin{tabular}{ccccc}
\hline \hline $\begin{array}{c}\text { Time } \\
\text { Scale }\end{array}$ & Range of Dates & $\begin{array}{c}\text { Sampling } \\
\text { Interval }\end{array}$ & $\begin{array}{c}\text { Number } \\
\text { of Points }\end{array}$ & $\begin{array}{c}\text { Temporal Frequency } \\
\text { Range (Hz) }\end{array}$ \\
\hline Short & 1997 May 22 00:14 - 1997 May 26 05:37 & $11.8 \mathrm{~min}$ & 512 & $5.7 \times 10^{-6}-7.0 \times 10^{-4}$ \\
Medium & 1997 Mar 16 00:01 - 1997 Jul 30 03:46 & $12.8 \mathrm{hr}$ & 256 & $1.7 \times 10^{-7}-1.1 \times 10^{-5}$ \\
Long & 1997 Mar 16 00:01 - 1998 Scp 12 16:06 & 4.27 days & 128 & $4.2 \times 10^{-8}-1.3 \times 10^{-6}$ \\
\hline
\end{tabular}


analysis is further restricted to the $2-10 \mathrm{keV}$ band, where the PCA is most sensitive and the systematic errors are best quantified.

The data reduction methods employed here are similar to those described by Nandra et al. (1998). In particular, binned light curves were initially extracted with $16 \mathrm{sec}$ time resolution from the STANDARD-2 data. Poor quality data were excluded using the criteria that: the Earth elevation angle was greater than 5 degree; the offset between the pointing position and the optical position of NGC 3516 was less than 0.01 degree and to exclude periods where the anti-coincidence rate in the propane layer of the PCUs was abnormal. This last criterion, used to identify anomalous clectron flares, is more fully described by Nandra et al. (1998). The relatively lax Earth elevation criterion was used to maximize the temporal coverage without significantly degrading the data quality. The $16 \mathrm{sec}$ sampled data were then rebinned to longer sampling intervals as discussed below. The light curve is shown in Figure 1.

\subsubsection{Background Model}

Background estimation represents the largest uncertainty for relatively weak sources such as NGC 3516. The three main components of the layer 1 background are those related to diffuse (sky) X-rays, a term related to many of the particle anti-coincidence rates and another that arises from decays induced by the South Atlantic Anomaly (SAA). Two background models, termed "SKY_VLE" and "L7," were considered. Only data using the latter model are used for the variability analysis, as the PCA team consider it more reliable, a conclusion that is confirmed below. The results obtained with each model are, however, sufficiently similar that they do not affect the conclusions presented in this paper. Both models are based on analysis of "sky" data from a number of pointings toward regions that are believed to be free of bright X-ray sources.

The "SKY_VLE" model estimates the particle background by relating it to the Very Large Event (VLE) rate of the PCA. About half of RXTE's orbits pass through the SAA each day, inducing background terms that decay exponentially. The SAA-related component was estimated by splitting the day into two halves. Orbits in the non-SAA half were assumed to be unaffected by previous passages, which is only valid if there are no long-term decays in the background. Under this assumption, background data were then used to estimate a component related to the VLE rate. The VLE model was then applied to the SAA orbits and the residual flux assumed to be that induced in the SAA. This flux was then correlated with satellite orbital parameters (BKGD_PHI and BKGD_THETA) to define the model. Then, the background for the current observations is estimated by taking the assumed spectral forms of the VLE and SAA-related components and combining them according to the observed VLE rates and positional parameters.

The "L7" model employs other PCA event rates that are directly related to both the particle and activation rates by a scaling factor. This leaves a residual induced term in the background that is modeled as an exponential decay with an e-folding time of $240 \mathrm{~min}$. The amplitude of 
this term is determined by integrating the rates from particle monitors on the High Energy X-ray Timing Experiment (HEXTE) on board RXTE, which are used to estimate the "dose" in the SAA.

We became aware of the importance of background subtraction during the campaign, and therefore began to accumulate offset data after (and sometimes before) the snapshots of NGC 3516 in a region of sky close by, but believed free of bright sources. These offset data were used to assess the systematic errors in the background subtraction methods. The L7 model gave the best subtraction of the offset data, and the background-subtracted offset data for that model is shown at the bottom of the top panel in Figure 1. Even for this best model, the mean is non-zero and the variance is larger than would be expected from statistical fluctuations. The mean and excess deviation are 0.87 and $0.39 \mathrm{c} / \mathrm{s}$ for the $\mathrm{L} 7$ model and 0.45 and $0.78 \mathrm{c} / \mathrm{s}$ for the SKY_VLE model. The lower excess variance shows that that the $\mathrm{L} 7$ is significantly better and that is why it was used in all subsequent analyses.

The corresponding section of the NGC 3516 light curve has an RMS deviation of $4.4 \mathrm{c} / \mathrm{s}$. The ratio of the squares (variances) is $\sim 125$, meaning that (in the worse case) typically less than $1 \%$ of the observed variability power is due to errors in the background model. The raw offset data have been corrected with the background-subtracted offset data, to see if there are any residual trends that have been poorly subtracted. The data showed no significant correlation, further strengthening confidence in the background subtraction. We therefore interpret the data under the assumption that the observed variability is dominated by intrinsic changes in the flux of NGC 3516, but caution that systematic errors in the background model could potentially cause subtle, low-level problems.

\subsection{Data Analysis}

The measurement of the PDS took two steps: First, the short, medium, and long time scale data were each separately made suitable for analysis and the individual PDS were measured, as

discussed in the next subsection. Then, the separate PDS were combined to produce a single PDS, as discussed in the second subsection.

\subsubsection{Construction of the Individual PDS}

The uneven sampling that made it so difficult for previous campaigns to estimate the PDS was much less of a problem here. The data were linearly interpolated (without adding Poisson noise) across the relatively rare data gaps, which generally spanned less than a few contiguous epochs because they tended to be short and spread quasi-randomly throughout the observation. This produced three light curves: (1) with 512 points sampled every $11.8 \mathrm{~min}$, (2) with 256 points sampled once every $12.8 \mathrm{hr}$, and (3) with 128 points sampled once every 4.3 days. Because the departures from the ephemeris were almost always small (typically only a few percent), 
interpolating and rebinning the data to an even grid would have made little difference (less than $0.3 \%$ on average, which is much less than the statistical or systematic errors). Thus, the ephemeris times and not the actual times were used in the analysis, with no interpolation.

PDS were then derived using standard methods from Numerical Recipes (Press et al. 1992) and the logarithms of both frequency and power were taken. The zero-power and next two lowest-frequency points of each PDS were ignored in the further analyses because they tend to be extremely noisy (see Press 1978 for details). The remaining points were binned every factor of two (0.3 in the logarithm), to reduce the noise and allow estimation of error bars. That means that the first binned point was derived by binning the two remaining lowest frequency points, the second by binning the next four points, etc.

The PDS were calculated using several window functions, $w_{j}$, to test for the effect of "red-noise leak" (e.g., Deeter \& Boynton 1982). These included the square window function

$$
w_{j}=1,
$$

the Welch window function,

$$
w_{j}=1-\left(\frac{j-\frac{1}{2} N}{\frac{1}{2} N}\right)^{2}
$$

the Hann window function,

$$
w_{j}=\frac{1}{2}\left[1-\cos \left(\frac{2 \pi j}{N}\right)\right],
$$

and the Bartlett window function,

$$
w_{j}=1-\left|\frac{j-\frac{1}{2} N}{\frac{1}{2} N}\right| .
$$

The individual PDS slopes differed only slightly depending on the window function. Results are presented in Figure 2 for the Bartlett window.

Power-law models were fitted to each PDS to estimate the slope, as shown in Table 2: column 2 gives the mean deviation of the data from the ephemeris, column 3 , the amount of data lost, column 4, the fitted slope (and uncertainty) of the power-law fit to each data set. The slope and uncertainty were measured from an unweighted, least-squares fit to the logarithmically binned PDS. The fractional variability $\left(F_{v a r}\right)$ is given in the last column. Unlike Edelson et al. (1996), this number is not corrected for intrinsic errors, because the uncertainties are dominated by systematic errors that have a different (and unknown) effect on different time scales, and in any event, the correction for these effects is small compared to the total observed value.

\subsubsection{Construction of the Combined PDS}

The short, medium, and long time scale PDS were then combined to form a single PDS spanning four decades of usable temporal frequency. As shown in Table 2, the PDS shows a highly 
Table 2. Variability Parameters

\begin{tabular}{ccccc}
\hline \hline $\begin{array}{c}\text { Time } \\
\text { Scale }\end{array}$ & $\begin{array}{c}\text { Mean Deviation } \\
\text { from Ephemeris }\end{array}$ & Data Lost & $\begin{array}{c}\text { Power-Law } \\
\text { Slope }(a)\end{array}$ & $\begin{array}{c}\text { Fractional } \\
\text { Variability }\left(F_{\text {var }}\right)\end{array}$ \\
\hline Short & - & $69 \mathrm{ksec}(19.1 \%)$ & $-1.74 \pm 0.12$ & $7.9 \%$ \\
Medium & $0.36 \mathrm{hr}(2.8 \%)$ & 10 points $(3.9 \%)$ & $-1.03 \pm 0.06$ & $30.1 \%$ \\
Long & $0.98 \mathrm{hr}(1.0 \%)$ & 2 points $(2.3 \%)$ & $-0.73 \pm 0.12$ & $31.6 \%$ \\
\hline
\end{tabular}

Table 3. PDS Fit Parameters

\begin{tabular}{lcc}
\hline \multicolumn{1}{c}{ Description } & Parameter & Value \\
\hline High frequency slope & $a$ & -1.76 \\
Cutoff frequency & $f_{c}$ & $4.14 \times 10^{-7} \mathrm{~Hz}$ \\
Cutoff time scale & $t_{c}\left(=1 / f_{c}\right)$ & 27 days \\
Normalization coefficients & $C_{1}$ & 8.54 \\
& $C_{2}$ & 354 \\
& $C_{3}$ & 646 \\
\hline
\end{tabular}


significant $(8 \sigma)$ systematic flattening from $a=-1.74 \pm 0.12$ to $-0.73 \pm 0.12$ as one goes from short to long time scales. These values were derived using the Bartlett window, but a similar difference of $(\Delta a \approx 1)$ was also seen for the other window functions. Because this same difference is seen for all window functions, we conclude that red-noise leak cannot be responsible for the change in PDS slope.

Thus, a more complex model than a single power-law was required to describe the PDS. All three PDS were simultaneously fitted with a model of a power-law that dominates at high frequencies, but cuts off to a slope of $a=0$ at very low temporal frequencies. We used the function

$$
P(f)=C_{1} /\left(1+f / f_{c}\right)^{a}
$$

where $P(f)$ is the fluctuation power at temporal frequency $f, a$ is the power-law slope at high temporal frequencies, $f_{c}$ is the "cutoff frequency," well below which the PDS flattens to a slope of zero, and $C_{1}$ is the normalization. Arbitrary, free relative normalizations $\left(C_{2}, C_{3}\right)$ were allowed between the three individual PDS. Including these two scaling factors, there are a total of five free parameters in the fit. The fit was performed by minimizing the Whittle negative log likelihood function with the result shown in Figure 3 and Table 3. The high-frequency slope is $a=-1.76$, similar to the slope derived using the high-frequency PDS alone. The cutoff frequency $f_{c}$ corresponds to a time scale of 27 days.

Although it is clear that the PDS is not consistent with a single power-law, the exact shape of the turnover is not well-constrained and the low-frequency behavior is undetermined. The fit assumed $a \rightarrow 0$ for $f \ll f_{c}$, but in fact it could be any slope with $a>-1$. (For $a \leq-1$, the total variability power would diverge.)

It is highly unlikely that the background problems discussed earlier could be responsible for the flattening in the PDS, because, as mentioned earlier, background errors contribute less than $1 \%$ of the total variability power. Furthermore, examination of Figure 1 shows that the background errors are correlated with each other; that is, their power is greater on long time scales than on short time scales. If anything, this would add more power at long time scales where the excursions are largest and relatively less at short time scales. This would steepen the PDS at long time scales, but this is the opposite of the flattening that is actually observed.

Finally, the $F_{v a r}$ values in Table 2 give independent evidence for a cutoff in the PDS at the longest time scales sampled. $F_{v a r}$ rises from $7.9 \%$ to $30.1 \%$ for short and medium time scale data taken over a period of 4.2 days and 4.5 months, respectively. However, it shows little further rise in the long time scale data, flattening out to $31.6 \%$ for sampling over 1.5 years. Again, visual examination of Figure 1 suggests that there is very little extra variability power on the longest time scales sampled. 


\section{Discussion}

We have obtained near-evenly sampled light curves of NGC 3516 over an unprecedented range of time scales. We have used these data to construct a PDS over 4 decades of temporal frequency. No sharp features were observed in the PDS. On short time scales, NGC 3516 exhibits "red-noise." The high-frequency slope is consistent with the mean values from EXOSAT of $a=-1.7$ and $a=-1.55$ derived by Green et al. (1993) and Lawrence \& Papadakis (1993), respectively. The extension of the PDS to lower frequencies afforded by the medium and long-time scale data has shown new results, however. The PDS is scen to progressively flatten at longer time scales and we interpret the overall PDS as possessing a characteristic variability time scale corresponding to the frequency of the turnover. Determining the variability time scale from these data is difficult, but our best estimate is $t_{c} \approx 1$ month.

\subsection{Physical Time Scales}

Here we state some characteristic time scales within the source, which may possibly be related to the observed flattening in the PDS. The fastest possible variability time scale for a coherent, isotropically-emitting region is the light-crossing time:

$$
t_{\mathrm{lc}}=0.011\left(M_{B H} / 10^{7} M_{\odot}\right)\left(R / 10 R_{S}\right) \text { day. }
$$

Here $M_{B H}$ is the black hole mass, $R$ is the distance from the center of mass and $R_{S}$ is the Schwarzschild radius. In a Comptonizing cloud this can be modified by scattering effects. The effect is negligible for low optical depth $(\tau \ll 1)$, but for $\tau>1$ the time scale is increased by approximately $\tau^{2}$.

From Kepler's Third Law, the matter orbital time scale is:

$$
t_{\text {orb }}=0.33\left(M_{B H} / 10^{7} M_{\odot}\right)\left(R / 10 R_{S}\right)^{3 / 2} \text { day }
$$

There are also several time scales related to the accretion disk. Specifically, for an $\alpha$-disk (Shakura \& Sunyaev 1973), relevant time scales are (Maraschi, Molendi \& Stella 1992; Treves, Maraschi \& Abramowicz 1988):

- Thermal:

$$
t_{\mathrm{th}}=5.3(\alpha / 0.01)^{-1}\left(M_{B H} / 10^{7} M_{\odot}\right)\left(R / 10 R_{S}\right)^{3 / 2} \mathrm{day}
$$

- Sound Crossing:

$$
t_{\text {sound }}=33(R / 100 H)\left(M_{B H} / 10^{7} M_{\odot}\right)\left(R / 10 R_{S}\right)^{3 / 2} \text { day }
$$

- Radial Drift/Viscous $(\dot{M})$ :

$$
t_{\text {drift }}=53000(R / H)^{2}(\alpha / 0.01)^{-1}\left(M_{B H} / 10^{7} M_{\odot}\right)\left(R / 10 R_{S}\right)^{3 / 2} \text { day } .
$$


Here, $\alpha$ is the viscosity parameter of the disk and $H$ is the scale height of the disk.

All of the time scales above depend on the mass of the black hole and the Distance from the center, in Schwarzschild radii, at which the X-rays are emitted. Black hole masses in local AGN have been estimated by various techniques and generally lie in the range $10^{6-9} \mathrm{M}_{\odot}$ (Ford et al. 1994; Miyoshi et al. 1995; Magorrian et al. 1998). The likely mass for NGC 3516 is in the range $10^{7-8} \mathrm{M}_{\odot}$, considering its luminosity. This implies that $t_{\mathrm{lc}}$ is much shorter than the turnover time scale $t_{\mathrm{c}}$. An even more compelling argument against the light-crossing time is that reverberation mapping indicates that the broad, optical-line region (BLR) in NGC 3516 is light-days to light-weeks across (Wanders et al. 1993). It is difficult envisage a scenario whereby the BLR is smaller than the continuum region that excited it. By similar arguments, the presence of a broad, iron K $\alpha$ line in NGC 3516 (Nandra et al. 1997a,b) indicates that the X-ray continuum-producing region lies inside the $100 R_{\mathrm{S}}$ within which the bulk of that emission line is produced. Wo therefore conclude that the turnover cannot be due purcly to light-travel time effects in the X-ray source.

\subsection{Model Implications}

The observed cutoff should be related to the fundamental physics that generates the variability. The process by which X-rays are produced in AGN is still not known, but typically models have concentrated on Compton upscattering of ultraviolet "seed" photons that probably arise in an accretion disk (e.g., Haardt \& Maraschi 1991, 1993; Zdziarski et al. 1994; Stern et al. 1995).

In such a Comptonizing plasma, which is posited to be the X-ray producing region in AGN, optical depth effects can smear out the variability, changing the power spectrum. For example, if the PDS was originally white noise, passage through such a plasma would introduce a cutoff, turning the high frequency PDS into "red noise" (e.g. Brainerd \& Lamb 1987; Kylafis \& Klimis 1987; Bussard et al. 1988; Lawrence \& Papadakis 1993). In such models, the turnover would be identified with the size of the scattering region, modified by optical depth effects. Identification of the observed turnover with such a process would require both a large region and a large optical depth. Even at the limits of plausible parameter space $\left(M_{B H} \approx 10^{8} \mathrm{M}_{\odot}\right.$ and $R \approx 100 R_{\mathrm{S}}$, however, an optical depth of order $\tau \sim 10$ would be required to match the observed value of $t_{\mathrm{c}}$. Current models of the X-ray spectra of AGN usually assume lower optical depths, and in fact most are optically thin (Haardt \& Maraschi 1991, 1993; Stern et al. 1995; Zdziarski et al. 1995). Some AGN emission models have optical depths as large as a few (e.g., Titarchuk \& Mastichidias 1994).

It is perhaps more likely, however, that the turnover corresponds to some different time scale. A specific model that has been proposed to explain the variability, the PDS and the variation of the PDS with luminosity is the so-called "bright spot" model of Bao and Abramowicz (1996). In this model, active regions on the surface of a rotating accretion disk produce the observed variability. In such a model the relevant turnover time scale could perhaps be identified with the 
$t_{\text {orb }}$, marginally consistent with the observed cutoff for the extremes of parameter space mentioned above if the emission is produced very far out in the disk.

Mineshige et al. (1994a,b) have presented a model in which the central regions of the accretion disk are in a "self-organized critical" (SOC) state. Their predicted PDS are qualitatively similar to those observed here, with PDS slopes of $a \approx 1.6$ at high frequencies and a turnover at longer time scales. In their model the central accretion disk exists in a near-critical state; when the mass density exceeds the critical value an "avalanche" of accretion occurs, emitting X-rays. It is these patchy, unstable regions that produce the X-rays, as well as the high frequency noise. The turnover time scale corresponds to $t_{\text {drift }}$ for the largest blobs. In a smoothly-accreting system, such as Mineshige et al. assume exists beyond the SOC region, $t_{\mathrm{drift}}$ is clearly too long to be associated with our turnover time scale. However, in the critical region in which the putative discrete blobs are present, the radial drift time scale can be much shorter, by a factor $\sim H / R$. Depending on the viscosity parameter, the accretion time scale can then approach the sound speed and match the observed cutoff.

Neither the "bright spot" nor the SOC models are clear about the acceleration mechanism or instability that causes the active regions to form, and it is quite plausible that the turnover is connected to these more fundamental mechanisms. Energy deposition into coronal hotspots might arise from thermal or acoustic instabilities in the disk whereby the turnover may be identified with $t_{\mathrm{th}}$ or $t_{\text {sound }}$, which are of the correct order. At this stage, it is most important that specific physical models of variability - which have been scarce to date - be constructed and PDS predicted for them. These can then be compared explicitly with these new data.

\subsection{Comparison with Galactic X-Ray Binaries}

The PDS of NGC 3516 looks remarkably like that of Cyg X-1 and other Galactic X-ray binaries (XRBs) in the "low" or hard state (see, e.g., Belloni \& Hasinger 1990; Miyamoto et al. 1992; van der Klis 1995). These sources exhibit a "red noise" spectrum at high frequencies with slopes $a$ between -1 and -2 . As observed in NGC 3516, they flatten to lower frequencies, with $a \approx 0$ and cutoff frequencies of order $0.1 \mathrm{~Hz}$ (XRBs can also exhibit a very low-frequency noise component, which shall be ignored for the purposes of this discussion.) The analogy between these sources and NGC 3516 is clear.

A simple prediction would be that the cutoff frequency would scale with physical size and therefore black hole mass, as one would expect, for example, for the light crossing or orbital time scales. In the absence of other specific models, one can relate the ratios of the black hole masses in Cyg X-1 and NGC 3516 by a simple scaling law. The cutoff frequency for Cyg X-1 varies between $f_{\mathrm{c}}=0.04-0.4 \mathrm{~Hz}$ (Belloni \& Hasinger 1990) while for NGC 3516, it appears to be of order $f_{\mathrm{c}} \approx 4 \times 10^{-7} \mathrm{~Hz}$. The ratio is $10^{5}-10^{6}$. The mass of the compact object in Cyg X-1 is thought to be of order $\sim 10 M_{\odot}$ by independent arguments (Herrero et al. 1995). Scaling the variability 
time scale as the mass $\left(M \propto R_{S} \propto t_{c}\right)$ yields a mass of $\sim 10^{6}-10^{7} M_{\odot}$ for NGC 3516 . This is to be compared with the estimates above for the black hole mass, i.e. similar to but perhaps a bit smaller than anticipated.

Of course, scalings and transformations of this kind are not necessarily valid unless one knows with which time scale the cutoff is identified. If, for example, the relevant quantity is one of the time scales in the accretion disk, several other parameters come into play which might be very different when comparing stellar and super-massive black holes. The luminosities of NGC 3516 and Cyg X-1 also scale as their cutoff frequencies, however, implying a similar Eddington ratio in the two cases.

The similarities in the PDS reinforce the idea that similar physical processes may produce the $\mathrm{X}$-rays in AGN and XRBs. The hard power-law in XRBs are thought to arise from Comptonization by hot electrons and of course similar models have been proposed for AGN. (See Van Paradijs 1998 for an excellent review of XRBs.) XRB models may deserve some consideration for AGN too. For example, Kazanas, Hua \& Titarchuk (1997) have proposed a model for XRBs in general and Cyg X-1 in particular, in which the general characteristics of the PDS can be produced by photon diffusion through a relatively large Comptonizing cloud, with variable density profile. As stated above, such Comptonization models require both large source regions and high optical depths. A prediction of this model is that there should be phase-dependent time lags between various energy bands in the X-rays (Hua, Kazanas \& Titarchuk 1997). This could be tested by searching the $R X T E$ data for lags between the hard and soft bands, although that will probably have to wait for further improvements in the background model and response matrix.

Along with the broad-band aperiodic variability, XRBs show quasi-periodic oscillations (QPO), pulsations and bursts. These latter properties are more easily related to the parameters of the central source (mass, magnetic field strength, etc.), but unfortunately, their existence in AGN remains controversial. Another interesting property, described for Cyg X-1 above, is that the cutoff frequency in XRBs is not constant. Prospects for measuring changes in $f_{\mathrm{c}}$ in AGN such as NGC 3516 are somewhat grim, given that it takes several years just to accumulate sufficient data to detect it.

\section{Conclusions}

By taking even sampling of the light curves of NGC 3516 on time scales that ranged from minutes to months, it has been possible to measure the PDS from $4 \times 10^{-8}$ to $7 \times 10^{-4} \mathrm{~Hz}$. This is more than twice the logarithmic dynamic range of the EXOSAT long-looks, which covered $\leq 2$ decades of usable temporal frequency near the high frequency end of this experiment.

The PDS of NGC 3516 is not consistent with a single power-law. The PDS slope changes from $a=-1.74$ at high temporal frequencies $\left(5 \times 10^{-6}-7 \times 10^{-4} \mathrm{~Hz}\right)$ to $a=-0.73$ at low temporal frequencies $\left(4 \times 10^{-8}-1.3 \times 10^{-6} \mathrm{~Hz}\right)$. Instead, the data are well-fitted by a high-frequency 
power-law slope of $a=-1.76$, with a cutoff at $f_{c} \approx 4 \times 10^{-7} \mathrm{~Hz}$. The PDS slope was assumed to go to a slope of zero for $f \ll f_{c}$. There is no evidence for strict or quasi-periodicity in the PDS.

The observed cutoff time scale is much too long to be associated with the light-crossing time for any reasonable range of source parameters. It is not terribly consistent with the rotating disk model of Bao \& Abramowicz (1996) unless the X-rays are produced in the very outer regions of the disk. It could be reconciled with Comptonization in a corona around an accretion disk (e.g., Haardt \& Maraschi 1993) if there are substantial optical depths effects (Kazanas et al. 1997). Another suggestion is that it represents the accretion time scale for blobs in a self-organized critical disk (Mineshige et al. 1994). In any event, measurement of the broad PDS and cutoff are basically new observational results, and at this stage, most models have not been developed to make strong predictions about them.

Perhaps the most striking result is the similarity to the PDS of Galactic XRBs. Besides having similar shapes, a direct scaling of mass with cutoff time scale (or luminosity) yields a black hole mass of $\sim 10^{6}-10^{7} M_{\odot}$ for NGC 3516. This may indicate that similar physical processes operate in both types of compact X-ray sources, spanning many orders of magnitude in luminosity. If so, we could hope to apply what has been learned about XRBs to help understand (the more difficult to study) AGN.

Although the PDS shows a clear change in slope, the actual shape of the low-frequency PDS is not well-determined. This can only be accomplished with data on even longer time scales, to sample even lower temporal frequencies than in this experiment. In fact, the 4.3 day sampling of NGC 3516 is continuing, and assuming that RXTE continues to operate until at least April 2000 , the PDS can be extended a factor of two lower in temporal frequency. Until these long time scales are sampled, it will not be possible to constrain the shape of the turnover. Indeed, characterizing the long time scale variability properties and cutoff frequency is likely be the lasting legacy of $R X T E$ for AGN, producing the first advance in our knowledge of PDS since EXOSAT and showing that the most interesting action is on the longest, not shortest accessible time scales.

As mentioned earlier, the near-perfect even sampling was achieved by the heroic effort, well above the call of duty, of Evan Smith of the RXTE SOC. The entire RXTE team put a great deal of effort into this project, without which it would not have been a success: Tess Jaffe and Gail Rorbach were instrumental in helping with the data reduction, Keith Jahoda, Dave Smith and Nick White helped with the background problems, Jean Swank scheduled the 1997 September-November observations that allowed smooth transition to the 4.3 day monitoring, and Alan Smale helped with a variety of issues. David Brillinger of the Berkeley Statistics Department also made a key contribution by explaining to us the statistics of combining data sampled on multiple time scales. Simon Vaughan and Mark Dixon helped with the data analysis. RE acknowledges financial support through NASA RXTE grant NAG 5-7315 and contract S-92507-Z, and KN acknowledges support through NASA grant NAG 5-7067 and the Universities Space Research Association. 


\section{REFERENCES}

Bao, G. \& Abramowicz, M. 1996, ApJ, 465, 646

Belloni, T. \& Hasinger, G. 1990, A\&A, 230, 103

Brainerd, J. \& Lamb, F. 1987, ApJ, 317, L33

Bussard, R. et al. 1988, ApJ, 327, 284

Deeter, J. \& Boynton, P. 1982, ApJ, 261, 337

Edelson, R. et al. 1996, ApJ, 470, 364

Edelson, R. et al. 1998, in preparation

Ford, H. et al. 1994, ApJ, 435, L27

George, I., Turner, T. J., Netzer, H., Nandra, K., Mushotzky, R., Yaqoob, T. 1998, ApJS, 114, 73

Ghosh, K. \& Soundararajaperumal, S. 1991, ApJ, 383, 574

Green, A., McHardy, I. \& Lehto, H. 1993, MNRAS, 265, 664

Haardt, \& Maraschi, L. 1991, ApJ, 380, L51

Haardt, \& Maraschi, L. 1993, ApJ, 413, 507

Herrero, A., Kudritzki, R., Gabler, R., Vilchez, J., Gabler, A. 1995, A\&A, 297, 556

Hua, X.-M., Kazanas, D., Titarchuk, L. 1997, ApJ, 482, L57

Iwasawa, K., Fabian, A., Brandt, W. N., Kunieda, H., Misaki, K., Reynolds, C. \& Rerashima, Y. 1998, MNRAS, 295L, 20

Jahoda, K., Swank, J., Giles, A., Stark, M., Strohmayer, T., Zhang, W., Morgan, E.H. 1996, EUV, X-ray and Gamma-ray Instrumentation for Space Astronomy VII, O. H. W. Sicgmund and M. A. Grummin, Eds., SPIE 2808, p. 59

Kazanas, D., Hua, X.-M., Titarchuk, L. 1997, ApJ, 480, 735

Kriss, G.A., et al. 1993, ApJ, 467, 629

Kolman, M. et al. 1993, ApJ, 403, 592

Koratkar, A. et al. 1998, BAAS, 192, 1607

Kylafis, N. \& Klimis, G. 1987, ApJ, 323, 678

Lawrence, A. \& Papadakis, I. 1993, ApJ, 414, L85

McHardy, I. in "Two Topics in X-ray Astronomy," Eds. H. Hunt \& B. Battrick, ESA SP-296 (ESA:Noordwijk), p. 1111

Maraschi, L., Molendi, S. \& Stella, L. 1992, MNRAS, 225, 27

Magorrian, J., et al. 1998, AJ, 115, 2285

Mineshige, S., Ouchi, N., Nishimoti, H. 1994, PASJ, 46, 97 
Mineshige, S., Takeuchi, M., Nishimoti, H. 1994, ApJ, 435, L125

Miyamoto, S., Kitamoto, S., Iga, S., Negoro, H., Terada, K. 1992, ApJ, 391, 21L

Miyoshi, M., Moran, J., Herrnstein, J., Greenhill, L., Nakai, N., Diamond, P., Inoue, M. 1995, Nat, 373,127

Nandra, K., George, I., Mushotzky, R., Turner, T. J., Yaqoob, T. 1997a, ApJ, 477, 602

Nandra, K., George, I., Mushotzky, R., Turner, T. J., Yaqoob, T. 1997b, MNRAS, 282, L7

Nandra, K., Clavel, J., Edelson, R., George, I., Malkan, M., Mushotzky, R., Peterson, B. \& Turner, T. J. 1998, ApJ, 505, 549

Nandra, K. \& Pounds, K. 1994, 268, 405

Papadakis, I. \& Lawrence, A. 1993, Nature, 361, 233

Papadakis, I. \& Lawrence, A. 1995, MNRAS, 272, 161

Papadakis, I. \& McHardy, I. 1995, MNRAS, 273, 923

Press, W. 1978, Comments on Astrophysics, 7, 103

Press, W. et al. 1992, "Numerical Recipes: The Art of Scientific Computing," 2nd Edition, (Cambridge University Press)

Rees, M. 1984, ARA\&A, 22, 472

Seyfert, K. 1943, ApJ, 97, 28

Shakura, N. \& Sunyaev, R. 1973, A\&A, 24, 337

Stern, B., Poutanen, J., Svensson, R., Sikora, M., Begelman, M. 1995, ApJ, 449, L13

Tagliaferri, G. 1996, ApJ, 465, 181

Terlevich, R. et al. 1995, MNRAS, 95, 272, 198

Titarchuk, L., Mastichiadis, A. 1994, ApJ, 433, L33

Treves, A., Maraschi, L., Abramowicz, M. 1988, PASP, 100, 427

van der Klis, M. 1995, in X-Ray Binaries, ed. W. Lewin, J. van Paradijs, \& van E. van den Heuvel (Cambridge: Cambridge Univ. Press), 252

Van Paradijs, J. 1998, in "The Many Faces of Neutron Stars," Eds. R. Buccheri et al. (Kulwar), in press, astro-ph $/ 9802177$

Voit, M., Shull, J. M. \& Begelman, M. 1987, ApJ, 316, 573

Wanders, I., et al. 1993, A\&A, 269, 39

Zdziarski, A., Fabian, A., Nandra, K., Celotti, A., Rees, M., Done, C., Coppi, P., Madejski, G. 1994, MNRAS, 269, L55 


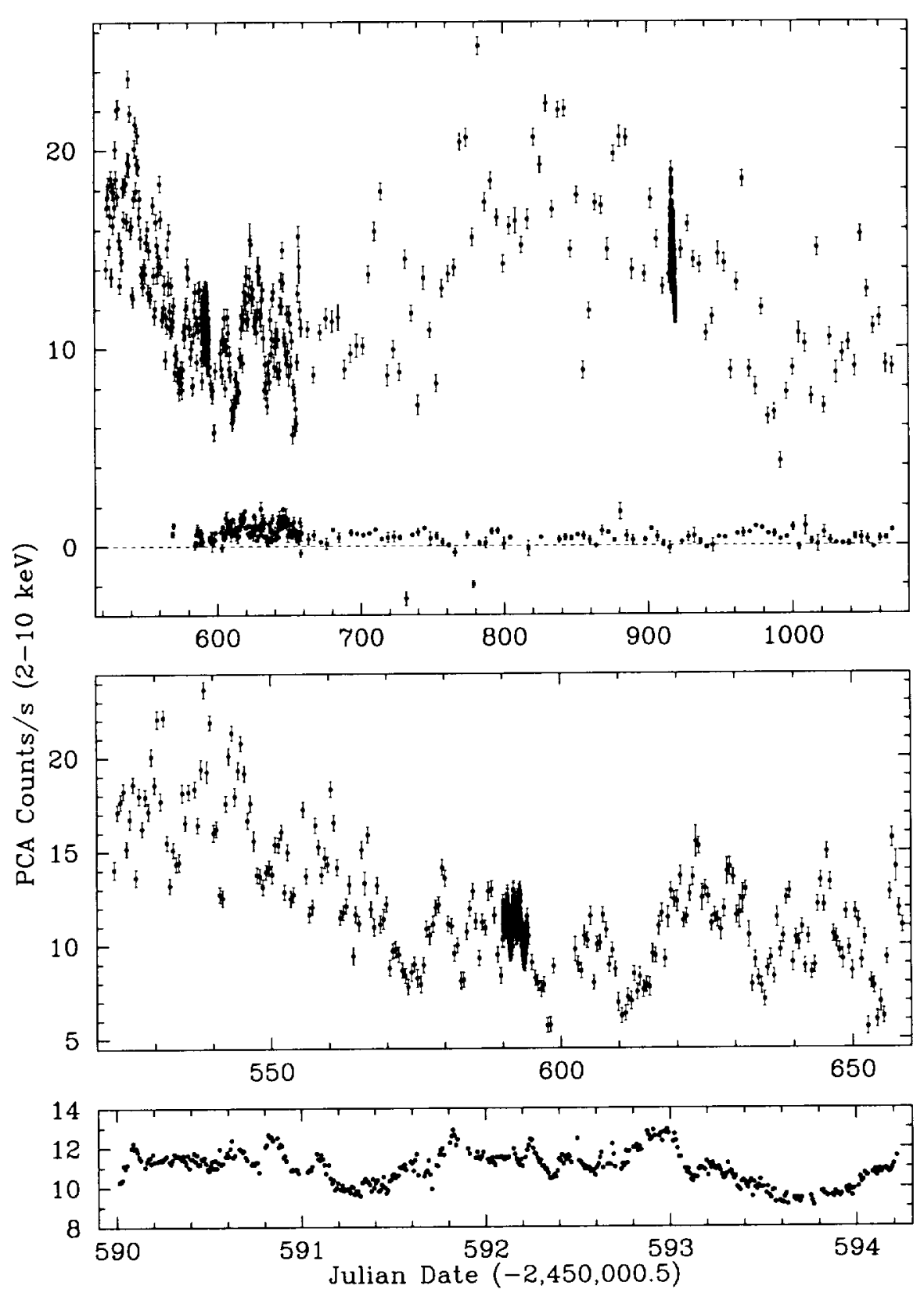

Fig. 1.- RXTE light curve of NGC 3516. The top panel covers the period 1997 March 16-1998 September 12 . Starting on 1997 July 31 , the light curve was sampled every $\sim 4.3$ days. The data at the top is the source light curve while those at the bottom $(-1$ to $+2 \mathrm{c} / \mathrm{s}$ ) are off-source (background pointings). The dense sampling around $\mathrm{MJD}=920$ was obtained for a simultaneous $H S T$ campaign, which will be discussed in a future paper (Edelson et al. 1998). The middle panel covers the period 1997 March 16 to July 30, during which time observations were made every $\sim 12.8 \mathrm{hr}$. The bottom panel shows the data during 1997 May 22 00:01-May 26 05:37, during which time the source was observed quasi-continuously, with interruptions only due to SAA passage and occasional conflicting time-critical observations, binned every $710 \mathrm{sec}$. The abscissa is the background-subtracted PCA $2-10 \mathrm{keV}$ Ll count rate. The error bars are a combination of statistical and systematic uncertainties 


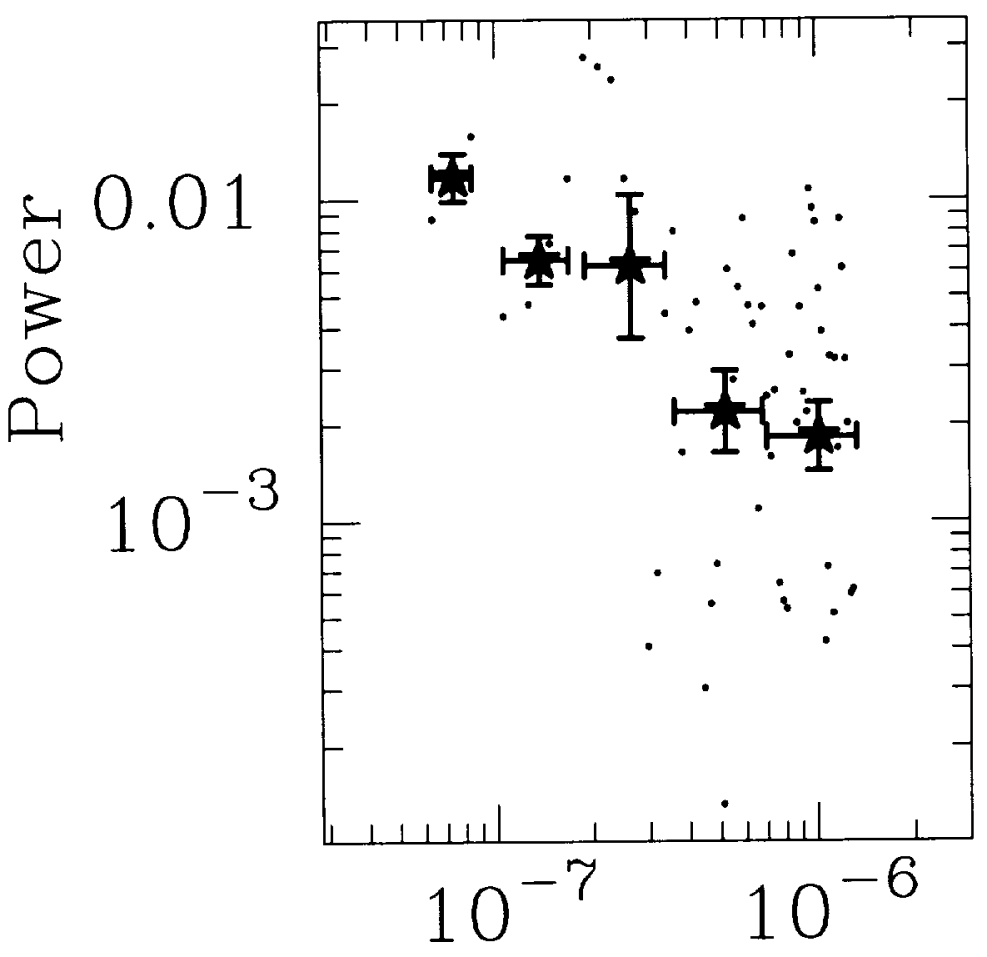

Temporal Frequency $(\mathrm{Hz})$

Fig. 2a.- PDS derived from the long time scale data. The first three points of the PDS have been suppressed as they represent the long-term power that is not accurately determined. The dots are the individual points in the PDS, while the large symbols are results of logarithmic binning. There is no evidence for periodicity, and the binned points were fitted with a power-law model, as discussed in the text. 


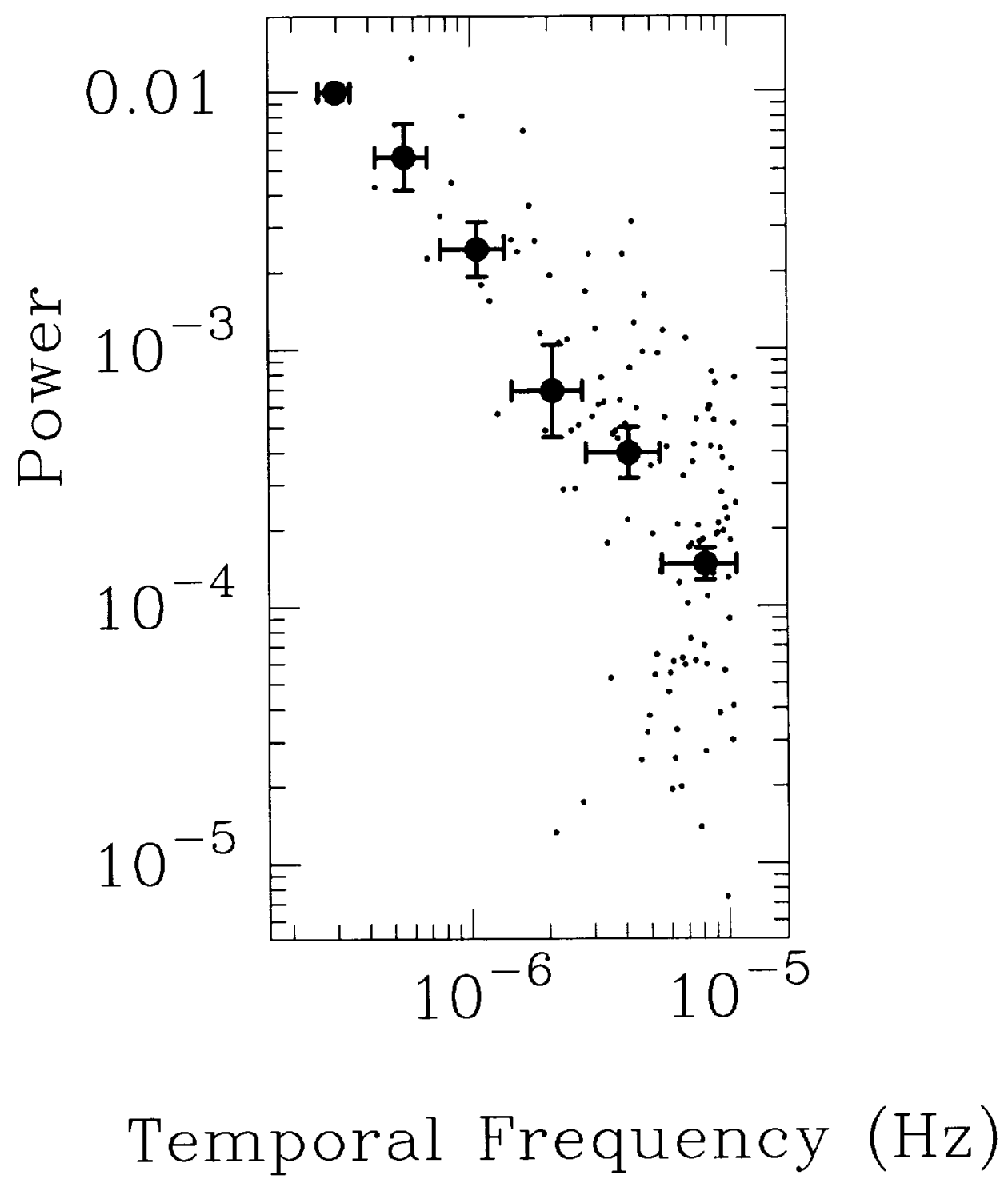

Fig. 2b.- Same as Figure 2a, except for the medium time scale data. 


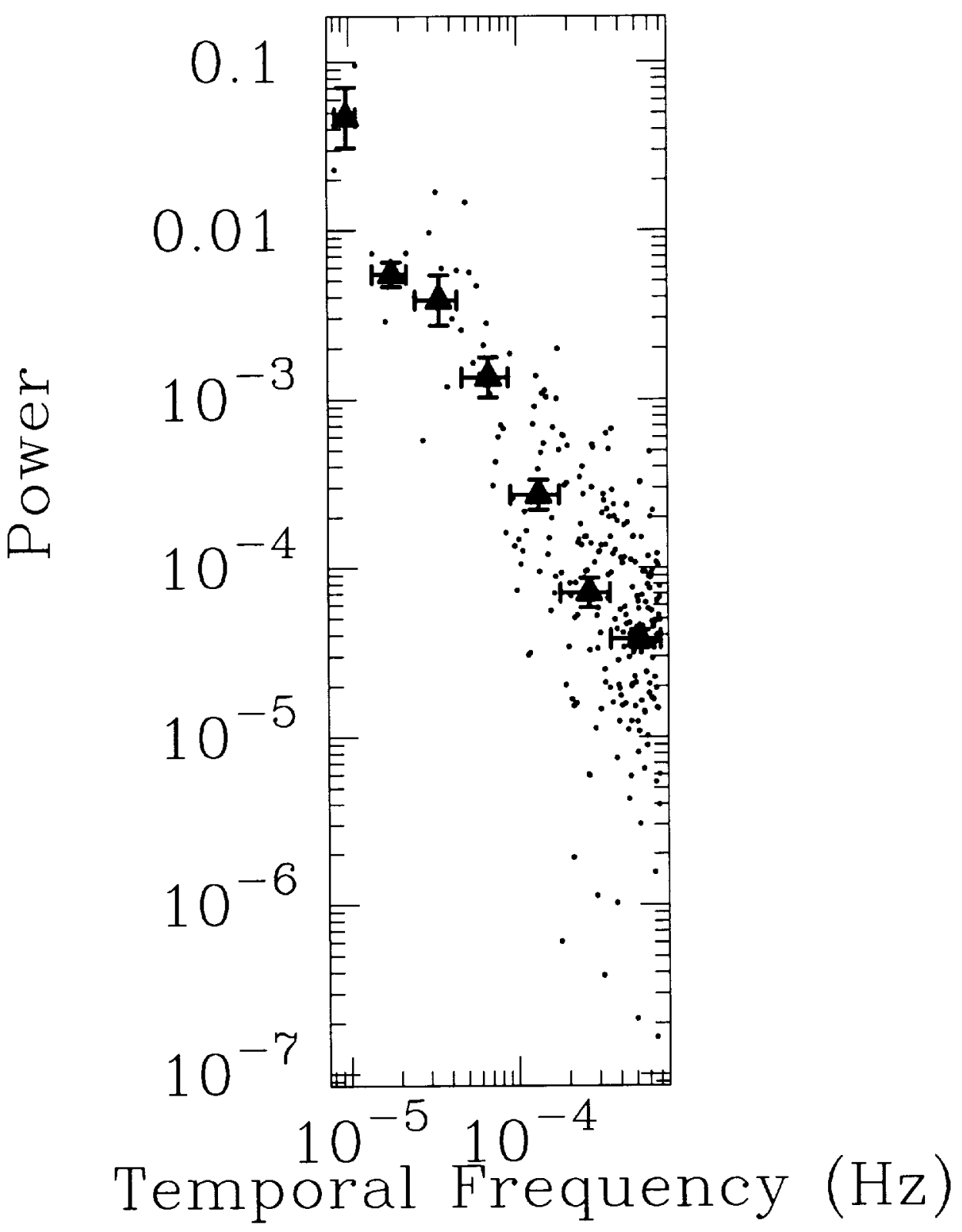

Fig. 2c.-- Same as Figure 2a, except for the short time scale data. 


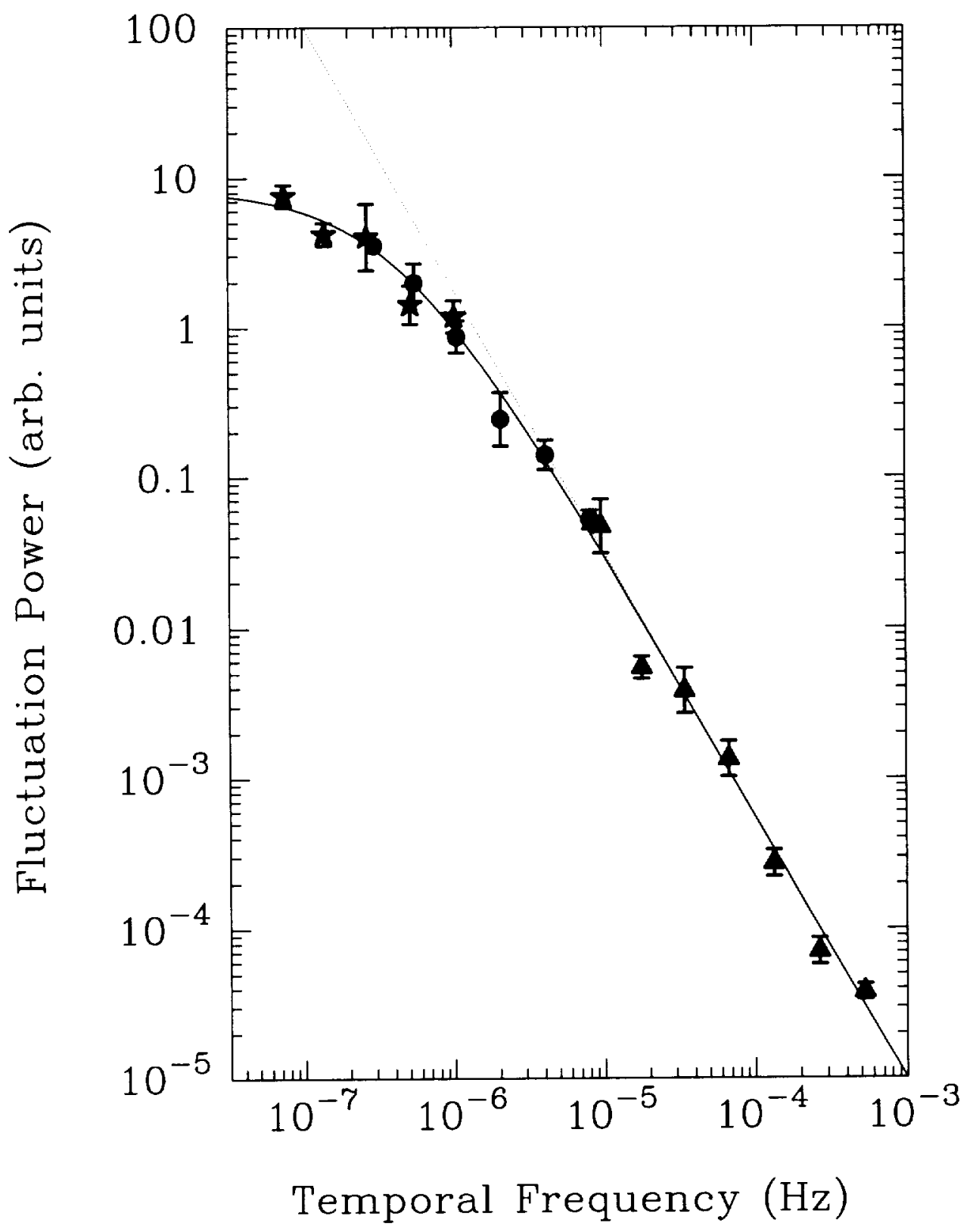

Fig. 3.- Broadband PDS of NGC 3516. The individual PDS in Fig. 2 were combined, scaled and fitted Simultaneously, as described in the text. The plot symbols follow the convention in Fig. 2. The PDS is not consistent with a single power-law; instead it had to be fitted by a power-law that flattened to low frequencies in order to obtain a reasonable fit. This yielded a cutoff frequency of $\sim 4 \times 10^{-7}$ Hz. corresnonding to $\sim 1$ month. 


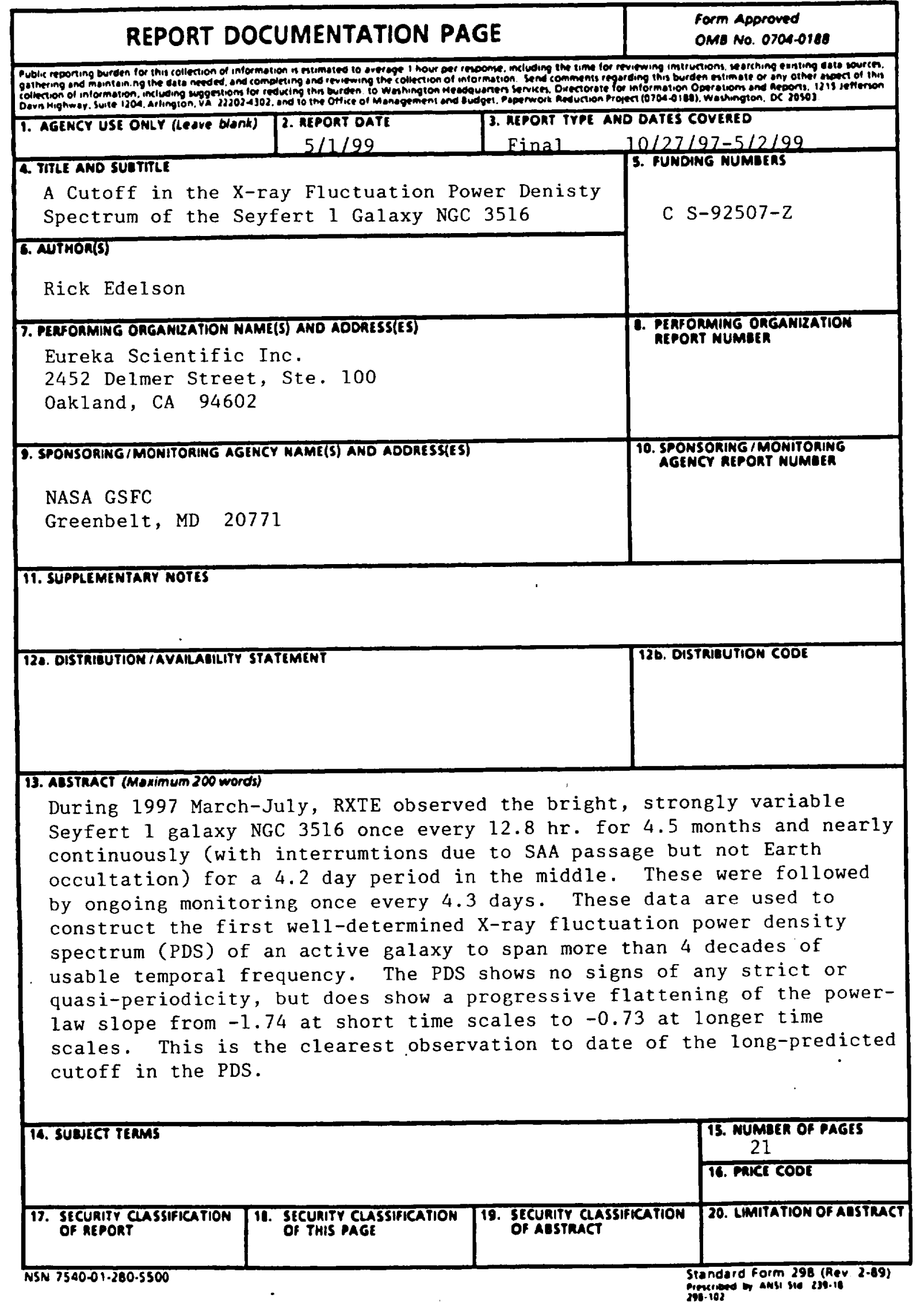

